\title{
Backward disequilibrium in elderly subjects
}

\author{
Patrick Manckoundia ${ }^{1,2}$ \\ France Mourey ${ }^{1,2}$ \\ Dominic Pérennou ${ }^{2,3}$ \\ Pierre Pfitzenmeyer ${ }^{1,2}$ \\ 'Department of Internal Medicine \\ and Geriatrics, University Hospital, \\ Dijon, France; ' ${ }^{2}$ INSERM/ERIT-M 0207 \\ Motricity-Plasticity University \\ of Burgundy, Dijon, France; \\ ${ }^{3}$ Department of Neurological \\ Rehabilitation, University Hospital, \\ Dijon, France
}

\begin{abstract}
Backward disequilibrium is observed frequently in daily clinical practice. However, there are no epidemiological data concerning this postural disorder. Defined by a posterior position of the centre of mass with respect to the base of support, backward disequilibrium is abnormal postural behavior, usually characterized by a posterior trunk tilt in standing and sitting positions, which predisposes subjects to backward falls. Many afflictions whether they are somatic (degenerative, ischemic and traumatic brain lesions), psychosomatic (psychomotor disadaptation syndrome, confinement to bed, nonuse situations) or psychological (depression) can cause backward disequilibrium. A vicious circle of falls, and loss of autonomy can arise and this is the main consequence of backward disequilibrium. Thus, in this paper, we review backward disequilibrium in elderly subjects with regard to the causes, consequences, assessment, and management.
\end{abstract}

Keywords: backward disequilibrium, balance, elderly subject, falls, posture

\section{Introduction}

The prevalence and the severity of posture and gait disorders increase with ageing (Salthouse and Somberg 1982; Maki et al 1994; Mourey et al 1998; Rankin et al 2000). Indeed, balance control is a complex sensori-motor function which requires the integration of vestibular, visual, and somesthetic information into the central nervous system (pyramidal, extrapyramidal, and cerebellar systems) in order to maintain antigravitar postures and to produce a suitable response to any balance perturbation (Horak and McPherson 1996). In addition, the quality of muscle and joints is important in the balance function (Alencar et al 2007).

Increased age is associated with impaired performance in vestibular, visual and somesthesic systems, a decline in the speed of information transmission and changes in the mode of information processing in the brain, all of which lead to balance and postural disorders (Brocklehurst et al 1982; Aniansson et al 1986; Woollacott 1993; Choy et al 2003; Yordanova et al 2004). Concerning the vestibule, it has been reported that the number of vestibular hair cells falls by $20 \%$ to $40 \%$ in healthy subjects aged 70 years and older, when compared to young subjects, and the number of vestibular nerve fibers has also been found to decrease with age (Alpini et al 2004). Regarding vision, decreased visual acuity, restriction of visual field, poor depth perception, losses in contrast sensitivity at the intermediate and high spatial frequencies have been found in elderly subjects (Alpini et al 2004). At the somesthesic level, various alterations in the morphology and functions of the peripheral nervous system are related to ageing. Elderly subjects also suffer from a loss of myelinated and unmyelinated nerve fibers associated with axonal atrophy. Aging also induces a decline in nerve conduction velocity and sensory discrimination (Verdu et al 2000). Moreover, the age-related decline in muscle and joint quality contributes to the proprioceptive abnormalities and also explains effector disorders found in elderly subjects. Indeed, with advancing age, the frequency of sarcopenia increases and the decrease in muscle mass is associated with the replacement of muscle fibers by fat and connective tissue 
(Lexell 1995; Alpini et al 2004; Crepaldi and Maggi 2005). Moreover, there is an increase in degenerative osteoarticular lesions and osteoporosis (Crepaldi and Maggi 2005). Independently of sensorimotor system, postural control is also enabled by spatial references such as the subjective vertical (Massion 1992). The subjective vertical (which can be haptic, postural, or visual) corresponds to an individual's perception of the vertical and is therefore different from the true or gravitational vertical (Manckoundia et al 2007a). Concerning gait, physiological ageing is associated with a reduction in the length and the height of step, an increase in dual foot contact time, and a resulting reduction in gait speed (Craik et al 1992; Alexander 1996).

The negative effects of certain chronic afflictions on posture and gait will compound those of physiological ageing. These chronic diseases may be neurological (Parkinson's disease or syndrome, the after-effects of stroke, neuropathy, normal pressure hydrocephalus and amyotrophic lateral sclerosis, for example) or osteoarticular (osteoarthritis of the lower limbs...), muscular, visual (cataract, age-related macular degeneration), vestibular and can be associated with certain geriatric syndromes such as psychomotor disadaptation syndrome (Knutsson 1972; Fisher 1982, Pfitzenmeyer et al 1999; Hausdorff 2000; Lee and Scudds 2003; Corriveau et al 2004; Manckoundia et al 2008).

These balance and postural disorders make elderly people vulnerable, accelerate functional decline and contribute to the loss of autonomy (Rankin et al 2000). Backward disequilibrium (BD) is a postural disorder which is characterized by a posterior position of the centre of mass with respect to the base of support in the standing and sitting position predisposing subjects to backward falls. This postural disorder is defined by a posterior body tilt (Mourey et al 2004). In addition, $\mathrm{BD}$ is associated with axial and limb rigidity. The pathophysiological mechanisms leading to BD behavior, however, have not yet been clearly identified. Nevertheless, as observed in patients with stroke, deterioration in the subjective vertical could be associated with the appearance of $\mathrm{BD}$. Indeed, the literature reports that the pusher behavior due to hemiplegia is associated with deterioration in the representation of the subjective vertical in the frontal plane (Lopez et al 1997; Pérennou et al 1998). A similar pathophysiological hypothesis may be involved in BD. To test this hypothesis we conducted a pilot study and found a direct correlation between the subjective vertical and the $\mathrm{BD}$ (Manckoundia et al 2007a). In this pilot study, the subjective vertical was assessed by measuring the postural vertical, which corresponds to the self-perception of the body's orientation in space and in the sagittal plane (Manckoundia et al 2007a). Moreover, BD could be related to accentuated postural tone of the extensor muscles due to a disorder in the excitability of the corresponding descending pathways (Porter and Lemon 1993), or to the fear of the void in front of the subject linked to the fear of falling in the context of post-fall syndrome (Murphy and Isaacs 1982; Pfitzenmeyer et al 1999; Mourey et al 2004).

Although the geriatrician can recognize $\mathrm{BD}$, which is not uncommon in daily clinical practice, there are no tools to evaluate the severity. Moreover, only a few authors have examined this postural disorder in elderly subjects (Mourey et al 2004) and these studies concern mainly Parkinsonian syndromes in the Anglo-Saxon literature (Visser et al 2003). Finally, there are no epidemiological data on BD.

Thus, in this paper, we review BD, a postural disorder that has severe consequences and can compromise functional autonomy in elderly subjects. We examine the etiologies, consequences, assessment and management. Indeed, the autonomy and functional well-being of elderly subjects suffering from BD could be improved by early diagnosis and management using basic interventions (Manckoundia et al 2007b).

\section{Afflictions and syndromes associated with backward disequilibrium}

Cortical and subcortical lesions of the central nervous system are the main abnormalities leading to $\mathrm{BD}$. There are reports of a positive correlation between cortical and subcortical cerebral lesions, identified on magnetic resonance imaging, and BD (Pfitzenmeyer et al 2002). Ischemic, degenerative and traumatic lesions of the cerebral trunk, the white matter, the frontal lobes, the gray matter and the cerebellum could induce BD in elderly subjects (van Wegen et al 2002). Ischemic and hemorrhagic strokes are the most frequent etiologies of BD as they are in nonidiopathic Parkinsonian syndromes (Visser et al 2003). In addition, the literature reports other subcortical abnormalities such as normal pressure hydrocephalus or amyotrophic lateral sclerosis, which could also be associated with BD (Desai and Swash 1999; Blomsterwall et al 2000).

Backward disequilibrium could also be due to an imbalance between the ankle extensor muscles and the ankle flexor muscles, because of hypertonia of extensor muscles, which could be caused by a peripheral neurological lesion (Porter and Lemon 1993). 
The main psychosomatic disorder associated with BD is psychomotor disadaptation syndrome and its acute form, the post-fall syndrome (Pfitzenmeyer et al 2001). Psychomotor disadaptation syndrome, which was first described by Gaudet and colleagues in 1986, is characterized by postural impairments, including BD, neurologic signs and psychological disorders (Mourey et al 2004). Several chronic diseases such as Parkinsonian syndromes, multiple system atrophy, progressive supranuclear palsy, Lewy body disease, Binswanger's disease, subcortical vascular lesions, normal pressure hydrocephalus, lymphomas of nervous system, meningoencephalitis, HIV infection, and depression may be associated with psychomotor disadaptation syndrome (Pfitzenmeyer 1999; Mourey et al 2004).

Finally, other situations such as confinement to bed and/ or depression could be associated with motor and postural disorders including BD (Mobily and Skemp Kelley 1991; Mourey et al 2004; Turcu et al 2004).

Afflictions associated with BD are summarized in Table 1.

\section{The main consequences of backward disequilibrium Falls and other physical consequences}

Elderly subjects suffering from BD have a high risk of falling backwards (Pfitzenmeyer et al 1999). Moreover, the diagnosis of BD is often made after a fall.

Falls are associated with an increase in morbi-mortality in the elderly (Wild et al 1981). Falls can cause trauma and have psychological and social consequences. Concerning trauma,

Table I Main afflictions associated with backward disequilibrium

Central nervous system lesions

Cortical vascular lesions

Subcortical vascular lesions

Ischemic stroke

Hemorrhagic stroke

Parkinsonian syndromes

Normal pressure hydrocephalus

Amyotrophic lateral sclerosis

Peripheral nervous system lesions

Peripheral neurological lesion inducing hypertonia of extensor muscles

Psychosomatic disorders

Psychomotor disadaptation syndrome

Confinement to bed

Depression hip fracture is the more dreaded injury (Rose and Maffulli 1999), and there is a risk of posttraumatic subdural hematoma (Noltie and Denham 1981). Fallers can also be affected by many other injuries (Tinetti et al 1995). These traumatic consequences are associated with a high risk of mortality (Tinetti et al 1995). Among the psychological consequences of falls, there is the fear of standing, walking, and falling (Gomez and Curcio 2007), all of which can exacerbate BD behavior and result in a vicious circle. The social consequences of falls include the loss of autonomy, institutionalization and the risk of becoming bedridden (Tinetti and Williams 1997). In addition, the loss of autonomy is associated with a decrease in physical activity leading to reduced muscle mass and functional decline which predispose the elderly to falls. Falls therefore have economic repercussions and represent a major public health problem (Tinetti et al 1994).

Regarding the consequences of falls, it is necessary for all health workers, especially physicians, involved in the management of elderly subjects to know how to recognize and manage syndromes and afflictions that could cause falls. In this context, BD, which is one of main risk factors of falls, must be detected.

Falls are a frequent consequence of moderate and severe forms of BD. However, elderly subjects suffering from mild form of BD show postural instability that does not necessarily result in falls. This, nevertheless, limits their daily activities, induces hypoactivity and may lead to the use of walking aids.

\section{Psychological and social consequences of backward disequilibrium}

Backward disequilibrium could have severe psychological consequences in elderly subjects even in the absence of falls. Indeed, BD is often associated with a loss of self-confidence during standing and walking. This could cause fear of standing, walking and falling as described in psychomotor disadaptation syndrome (Mourey et al 2004; Manckoundia et al 2007b). This fear of standing, walking and falling can appear even in the absence of a history of falls (Legters 2002). The progressive loss of autonomy is the logical outcome of both loss of self-confidence during standing/walking, and fear of standing. BD and its motor and psychological consequences lead to postural disadaptation which in turn leads to dependence.

Finally, BD can bring about depression mostly because of the fear of walking and falling, loss of independence and decreased of quality of life. Indeed, elderly people who become aware of the deterioration in their physical 
capacities will lose self-confidence and self-esteem, develop a sense of insecurity and become withdrawn. As a result they lose motivation and restrict physical and social activities.

\section{Assessment of backward disequilibrium}

Among the tests to assess balance and gait in elderly subjects, only the Minimum Motor Test includes items for a qualitative analysis of BD (Mourey et al 2005). The other usual tests, such as the Tinetti test (Tinetti 1986), the Timed Up and Go test (Podsiadlo and Richardson 1991) or the Berg Balance Scale (Berg et al 1992), are not able to diagnose $\mathrm{BD}$. Indeed, these tests are only suitable to evaluate the consequences of BD on certain daily tasks such as sit-to-stand, back-to-sit, maintaining a standing position or walking. Given the absence of a specific tool to assess BD, diagnosis remains only qualitative, based on observations of elderly subjects in the sagittal plane, in the sitting position as well as in the static and dynamic standing position. However, a quality assessment of this kind of postural disorder requires a quantitative scale.

\section{Management of backward disequilibrium}

The management of elderly subjects suffering from BD has to be multidisciplinary, associating different categories of health professionals including physicians, physiotherapists, psychologists, nurses, and nurse's aides. In addition, the management of BD must begin quickly, immediately after making the diagnosis, which is based on clinical observation.

The medical management consists of the search for and the treatment of possible causes of the BD. The physician must therefore make a detailed examination, which could be completed by targeted biological analyses or radiological examinations.

There are few reports concerning the rehabilitation of frail patients showing major limitations in motor performance. The association of strength training and progressive functional training improves the physical capacities in frail geriatric patients (Hauer et al 2001). A recent review of the literature shows that progressive resistance training alone is not an effective therapy for balance control (Orr et al 2008). Improving the dynamic and static control of the centre of mass is the main objective of physiotherapy. Practicing turning over in bed is introduced very early in the rehabilitation program in order to restore autonomy in bed and the capacity to achieve transfers. Then, balance training is implemented in static and dynamic positions. It is advisable to start balance rehabilitation using intrinsic exercises first, followed by extrinsic exercises in the sitting position as well as in the standing position. The forward projection of the trunk and the stabilization of the head in space are developed in order to make the sit-to-stand easier. Indeed, the sit-to-stand is a determinant stage of functional rehabilitation. In the standing position, the use of a removable heelpiece could help to reduce BD at the beginning of the physiotherapy. The rehabilitation of walking using walking aids must be started as soon as possible. In addition, subjects suffering from $\mathrm{BD}$ have to be trained to rise from the floor. Rehabilitation could be limited because these patients tire easily. The physiotherapist, therefore has to control the heart rate and the breathing frequency (Brondel et al 2005). Balance/functional training should last 30 minutes per training session. The choice of exercise must be personalized according to the postural abilities of the subject.

In parallel with rehabilitation by the physiotherapist, nurses and nurse's aides have to stimulate elderly patients suffering from BD through the activities of everyday life, but in doing so they must avoid pulling on the patient's upper limbs during transfers.

Finally, the psychologist must reassure and motivate elderly subjects suffering from BD and not overdramatize the situation to help them regain self-confidence. Besides, the psychologist must help elderly subjects suffering from BD to overcome disafferentation, fear of standing and walking as well as fear of falling (Manckoundia et al 2007b).

\section{Conclusion}

In this paper, we have reviewed current knowledge about BD in the elderly. Although it has been known by physicians for several decades, $\mathrm{BD}$, which could have serious physical, psychological or social consequences, has rarely been studied in the literature. The few published studies on BD concern Parkinsonian syndromes. The causes of BD are often mixed and associate somatic and psychological pathologies among which there is post-fall syndrome. The management of BD must be interdisciplinary (involving physicians, physiotherapists, psychologists, nurses, nurses' aides). Physiotherapy, which is a major component of the management strategy, must be started early and adjusted to all of the situations of everyday life. Future studies have to provide data on the prevalence and incidence of $\mathrm{BD}$. 


\section{Disclosure}

The authors report no conflicts of interest in this work.

\section{References}

Alencar MA, Arantes PMM, Dias JMD, et al. 2007. Muscular function and functional mobility of faller and non-faller elderly women with osteoarthritis of the knee. Braz J Med Biol Res, 40:277-83.

Alexander NB. 1996. Gait disorders in older adults. $J$ Am Geriatr Soc, 44:434-51.

Alpini D, Cesarani A, Fraschini F, et al. 2004 Aging and vestibular system: specific tests and role of melatonin in cognitive involvement. Arch Gerontol Geriatr, 9(Suppl):13-25.

Aniansson A, Hedberg M, Henning GB, et al. 1986. Muscle morphology, enzymatic activity, and muscle strength in elderly men: a follow-up study. Muscle Nerve, 9:585-91.

Berg K, Wood-Dauphinee SL, Williams JI, et al. 1992. Measuring balance in the elderly: validation of an instrument. Can J Public Health, 83(suppl 2):S7-11.

Blomsterwall E, Svantesson U, Carlsson U, et al. 2000. Postural disturbance in patients with normal pressure hydrocephalus. Acta Neurol Scand, 102:284-91.

Brocklehurst JC, Robertson D, James-Groom P. 1982. Clinical correlates of sway in old age-sensory modalities. Age Ageing, 11:1-10.

Brondel L, Mourey F, Mischis-Troussard C, et al. 2005. Energetic cost and cardiorespiratory adaptation in Get-Up-and-Go test in frail elderly women with postural abnormalities and in controls. J Gerontol A Biol Sci Med Sci, 60:98-103.

Choy NL, Brauer S, Nitz J. 2003. Changes in postural stability in women aged 20 to 80 years. $J$ Gerontol A Biol Sci Med Sci, 58A:M525-30.

Corriveau H, Hébert R, Raîche M, Prince F. 2004. Evaluation of postural stability in the elderly with stroke. Arch Phys Med Rehabil, 85:1095-101.

Craik RL, Dutterer LA, Riggle CL. 1992. Comparaison of walking patterns between young and older men. In: Woollacott M, Horak F (ed). Posture and Gait: control mechanisms. Oregon: University Oregon Books. pp. 243-6.

Crepaldi G, Maggi S. 2005. Sarcopenia and osteoporosis: A hazardous duet. $J$ Endocrinol Invest, 28(10 Suppl):66-8.

Desai J, Swash M. 1999. Extrapyramidal involvement in amyotrophic lateral sclerosis: backward falls and retropulsion. J Neurol Neurosurg Psychiatry, 67:214-16.

Fisher CM. 1982. Hydrocephalus as a cause of disturbances of gait in the elderly. Neurology, 32:1358-63.

Gomez F, Curcio CL. 2007. The development of a fear of falling interdisciplinary intervention program. Clin Interv Aging, 2:661-7.

Hausdorff JM, Lertratanakul A, Cudkowicz ME, et al. 2000. Dynamic markers of altered gait rhythm in amyotrophic lateral sclerosis. $J$ Appl Physiol, 88:2045-53.

Hauer K, Rost B, Rütschle K, et al. 2001. Exercise training for rehabilitation and secondary prevention of falls in geriatric patients with a history of injurious falls. $J$ Am Geriatr Soc, 49:10-20.

Horak FB, McPherson JM. 1996. Postural orientation and equilibrium. In: Rowell LB, Shepherd JT, (ed). Handbook of physiology. New York: Oxford University Press. pp. 255-92.

Knutsson E. 1972. An analysis of parkinsonian gait. Brain, 95:475-86.

Lee HKM, Scudds RJ. 2003. Comparison of balance in older people with and without visual impairment. Age Ageing, 32:643-9.

Legters K. 2002. Fear of falling. Phys Ther, 82:264-72.

Lexell J. 1995. Human aging, muscle mass, and fiber type composition. J Gerontol A Biol Sci Med Sci, 50(Spec No):11-6.

Lopez I, Honrubia V, Baloh RW. 1997. Aging and the human vestibular nucleus. $J$ Vest Res, 7:77-85.

Maki BE, Holliday PJ, Topper AK. 1994. A prospective study of postural balance and risk of falling in an ambulatory and independent elderly population. J Gerontol, 49:M72-84.
Manckoundia P, Mourey F, Pfitzenmeyer P, et al. 2007a. Is backward disequilibrium in the elderly caused by an abnormal perception of verticality? A pilot study. Clin Neurophysiol, 118:786-93.

Manckoundia P, Gerbault N, Mourey F, et al. 2007b. Mutidisciplinary management in geriatric day hospital is beneficial for elderly fallers: a prospective study of 28 cases. Arch Gerontol Geriatr, 44:61-70.

Manckoundia P, Thomas F, Buatois S, et al. 2008. Impact of clinical, psychological and social factors on decreased Tinetti test score in community-living elderly subjects: A prospective study with two years follow-up. Med Sci Monit, 14:CR316-22.

Massion J. 1992. Movement, posture and equilibrium: interaction and coordination. Prog Neurobiol, 38:35-56.

Mobily PR, Skemp Kelley LS. 1991. Iatrogenesis in the elderly. Factors of immobility. J Gerontol Nurs, 17:5-11.

Mourey F, Camus A, d'Athis P, et al. 2005. Mini motor test: a clinical test for rehabilitation of patients showing psychomotor disadaptation syndrome (PDS). Arch Gerontol Geriatr, 40:201-11.

Mourey F, Manckoundia P, Martin-Arveux I, et al. 2004. Psychomotor disadaptation syndrome. A new clinical entity in geriatric patients. Geriatrics, 59:20-4.

Mourey F, Pozzo T, Rouhier-Marcer I, et al. 1998. A kinematic comparison between elderly and young subjects standing up from and sitting down in a chair. Age Ageing, 27:137-46.

Murphy J, Isaacs B. 1982. The post-fall syndrome. A study of 36 elderly patients. Gerontology, 28:265-70.

Noltie K, Denham MJ. 1981. Subdural haematoma in the elderly. Age Ageing, 10:241-6.

Orr R, Raymond J, Fiatarone Singh M. 2008. Efficacy of progressive resistance training on balance performance in older adults: a systematic review of randomized controlled trials. Sports Med, 38:317-43.

Pérennou DA, Amblard B, Leblond C, et al. 1998. Biased postural vertical in humans with hemispheric cerebral lesions. Neurosci Lett, 252:75-8.

Pfitzenmeyer P, Martin-Hunyadi C, Mourey F, et al. 2002. Cardiovascular characteristics and cerebral CT findings in elderly subjects with psychomotor disadaptation syndrome. Aging Clin Exp Res, 14:100-7.

Pfitzenmeyer P, Mourey F, Mischis-Troussard C, et al. 2001. Rehabilatation of serious postural insufficiency after falling in very elderly subjects. Arch Gerontol Geriatr, 33:211-18.

Pfitzenmeyer P, Mourey F, Tavernier B, et al. 1999. Psychomotor desadaptation syndrome. Arch Gerontol Geriatr, 28:217-25.

Podsiadlo D, Richardson S. 1991. The timed "Up and Go": a test of basic functional mobility for frail elderly persons. J Am Geriatr Soc, $39: 142-8$.

Porter R, Lemon R. 1993. Corticospinal function and voluntary movement. Oxford: Clarendon Press.

Rankin JK, Woollacott MH, Shumway-Cook A, et al. 2000. Cognitive influence on postural stability: a neuromuscular analysis in young and older adults. J Gerontol A Biol Sci Med Sci, 55A:M112-19.

Rose S, Maffulli N. 1999. Hip fractures. An epidemiological review. Bull Hosp Jt Dis, 58:197-201.

Salthouse TA, Somberg BL. 1982. Time-accuracy relationships in young and old adults. J Gerontol, 37:349-53.

Tinetti ME. 1986. Performance - oriented assessment of mobility problems in elderly patients. $J$ Am Geriatr Soc, 34:119-26.

Tinetti ME, Baker DI, McAvay G, et al. 1994. A multifactorial intervention to reduce the risk of falling among elderly people living in the community. N Engl J Med, 331:821-7.

Tinetti M, Doucette J, Claus E, et al. 1995. Risk factors for serious injury during falls by older persons in the community. J Am Geriatr Soc, 43:1214-21.

Tinetti ME, Williams CS. 1997. Falls, injuries, and the risk of admission to a nursing home. N Engl J Med, 337:1279-84.

Turcu A, Toubin S, Mourey F, et al. 2004. Falls and depression in older people. Gerontology, 50:303-8. 
van Wegen EE, van Emmerik RE, Riccio GE. 2002. Postural orientation: age-related changes in variability and time-to-boundary. Hum Mov Sci, 21:61-84.

Verdu E, Ceballos D, Vilches JJ et al. 2000. Influence of aging on peripheral nerve function and regeneration. J Peripher Nerv Syst, 5:191-208.

Visser M, Marinus J, Bloem BR, et al. 2003. Clinical tests for the evaluation of postural instability in patients with Parkinson's disease. Arch Phys Med Rehabil, 84:1669-74.
Wild D, Nayak USL, Isaacs B. 1981. How dangerous are falls in old people at home? Br Med J, 282:266-8.

Woollacott MH. 1993. Age-related changes in posture and movement. $J$ Gerontol, 48(Spec. $\left.\mathrm{N}^{\circ}\right): 56-60$.

Yordanova J, Kolev V, Hohnsbein J, et al. 2004. Sensorimotor slowing with ageing is mediated by a functional dysregulation of motor-generation processes: evidence from high-resolution event-related potentials. Brain, 127:351-62. 